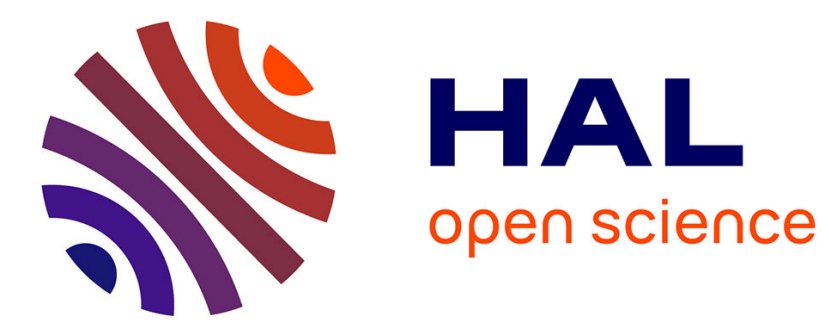

\title{
Evidence for magnon BEC in superfluid 3He-A
}

Pierre Hunger, Yuriy M. Bunkov, Eddy Collin, Henri Godfrin

\section{To cite this version:}

Pierre Hunger, Yuriy M. Bunkov, Eddy Collin, Henri Godfrin. Evidence for magnon BEC in superfluid 3He-A. Journal of Low Temperature Physics, 2010, 158 (1), pp.129. 10.1007/s10909-009-9970-3 . hal00917395

\section{HAL Id: hal-00917395 \\ https://hal.science/hal-00917395}

Submitted on 13 Dec 2013

HAL is a multi-disciplinary open access archive for the deposit and dissemination of scientific research documents, whether they are published or not. The documents may come from teaching and research institutions in France or abroad, or from public or private research centers.
L'archive ouverte pluridisciplinaire HAL, est destinée au dépôt et à la diffusion de documents scientifiques de niveau recherche, publiés ou non, émanant des établissements d'enseignement et de recherche français ou étrangers, des laboratoires publics ou privés. 


\title{
Evidence for magnon BEC in superfluid ${ }^{3} \mathrm{He}-\mathrm{A}$
}

\author{
P. Hunger, Yu.M. Bunkov, E. Collin and \\ H. Godfrin
}

Received: date / Accepted: date

\begin{abstract}
The phenomenon of phase-coherent precession of magnetization in superfluid ${ }^{3} \mathrm{He}$ and the related effects of spin superfluidity are based on the true Bose-Einstein condensation of magnons. Several different states of coherent precession have been observed in ${ }^{3} \mathrm{He}-\mathrm{B}$ : homogeneously precessing domain (HPD); persistent signal formed by $Q$-balls at very low temperatures; coherent precession with fractional magnetization; and two new modes of the coherent precession in compressed aerogel. Here we demonstrate the evidence of magnons Bose-Einstein condensation in ${ }^{3} \mathrm{He}-\mathrm{A}$ in a compressed aerogel.
\end{abstract}

Keywords First keyword · Second keyword · More

PACS 67.57.Fg $\cdot 05.45 . \mathrm{Yv} \cdot 11.27 .+\mathrm{d}$

\section{Introduction}

The discovery [?] and detailed investigations of the phase-coherent precession of magnetization in superfluid ${ }^{3} \mathrm{He}-\mathrm{B}$ generated a search for similar phenomena in other systems. The interest to these phenomena particularly increased after their interpretation as a magnon Bose-Einstein condensation [?]. Superfluid ${ }^{3} \mathrm{He}-\mathrm{A}$ could be an other system where this phenomenon can be observed. However, it was found that under typical conditions the coherent precession in ${ }^{3} \mathrm{He}-\mathrm{A}$ is unstable [?] because of the convex shape of the spin-orbit energy potential as function of magnetization [?,?]. It was suggested that the shape of this potential can be inverted and thus the coherent precession can be stabilized if the orbital momentum of Cooper pairs in ${ }^{3} \mathrm{He}-\mathrm{A}$ is oriented along the applied magnetic field [?]. Recently such orientation has been reached for ${ }^{3} \mathrm{He}-\mathrm{A}$ immersed in axially squeezed aerogel [?]. It was also found that in conditions of permanent RF pumping, all magnetization can be deflected on a large angle and precess at the frequency and phase of the RF field [?]. This phenomenon corresponds well to the theory of coherent precession of magnetization [?], and particularly to the theory of Coherent precession in ${ }^{3} \mathrm{He}-\mathrm{A}[?]$. Indeed, to prove the formation of a magnon BEC one should

MCBT, Institut Néel, CNRS/UJF, Grenoble, 38042, France

E-mail: yuriy.bunkov@grenoble.cnrs.fr 
demonstrate the stability of phase coherent precession without RF pumping. To confirm the formation of a self-consistent Coherent Precession State (CPS), we performed in Grenoble pulsed NMR experiments with ${ }^{3} \mathrm{He}-\mathrm{A}$ immersed in a squeezed aerogel.

\section{Experimental conditions}

We used a stycast cell in a form of vertical cylinder, in which aerogel (98\% porosity, produced by N. Mulders) was vertically pressed of about $10 \%$ of its length in order to create an uniaxial anisotropy. It was then put in the experimental space of a nuclear demagnetization refrigerator filled with ${ }^{3} \mathrm{He}$ and studied by the means of continuous wave and pulse NMR. The temperature of our experiment is given by the dissipation of a tantalum vibrating wire in bulk ${ }^{3} \mathrm{He}$. We filled the experimental cell by an amount of ${ }^{4} \mathrm{He}$ sufficient to cover the aerogel strands and avoid any signal due to solid layers of adsorbed ${ }^{3}$ He. Pressure in all data exposed below was 29.3 bars and NMR fields were either $87.6 \mathrm{mT}$ or $72.5 \mathrm{mT}$, corresponding to respective frequencies of $2.84 \mathrm{MHz}$ and $2.35 \mathrm{MHz}$.

The NMR excitation field $H_{1}$ was measured directly for the pulse NMR experiment. In normal phase, the amplitude of the free induction signal is proportional to the sine of the deflection angle given by $\theta=\gamma_{3} \mathrm{He}_{\mathrm{H}} \mathrm{H}$. In our experiment, a deflection angle of $\pi$ corresponds to 44 pulses at $2.352 \mathrm{MHz}$ which gives an excitation field of $8.2 \times 10^{-4} \mathrm{mT}$. Knowing this relation between field and current in the coil, one can easily determine the field for the continuous wave experiments which varies between $0.1 \mu \mathrm{T}$ for small drive experiments and $5 \mu \mathrm{T}$ for our highest drive experiments.

The superfluid transition temperature in our sample is $T_{c a}=1.77 \mathrm{mK}$ and the transition from A-like to B-like phase occurs at $T_{a b}=1.32 \mathrm{mK}$. This transition occurs at lower temperatures than usually observed in aerogel. It may be an effect of the highest density of our pressed aerogel sample.

\section{Coherently precessing state in ${ }^{3} \mathrm{He}-\mathrm{A}$}

The nuclear magnetic part of superfluidity of ${ }^{3} \mathrm{He}$ corresponds to very original nuclear antiferromagnetic states. The dipole-dipole interaction between the nuclei is of the order of 1 oersted and depends on the orientation of the spin and orbit parts of coherent wave function. This interaction manifests itself by a frequency shift of the magnetic resonance which depends on the relative orientation of the spin and orbit parts of wave function of ${ }^{3} \mathrm{He}$. If one deflects the magnetization from its statical orientation along the magnetic field, the frequency shift changes. Consequently, the magnetic resonance has non-linear properties. In Fig.?? are shown the frequency shift of ${ }^{3} \mathrm{He}-\mathrm{A}$ NMR dependence on the angle of deflection of magnetization for a usual case, when orbital momentum $\mathbf{L}$ is fixed perpendicular to a magnetic field $\mathbf{H}$ (usual conditions for NMR in bulk ${ }^{3} \mathrm{He}-\mathrm{A}$ ), and for the case when $\mathbf{L}$ is reoriented along to magnetic field. It was shown recently [?,?] that squeezing aerogel fixes the orientation of $\mathbf{L}$ along the direction of squeezing. In current experiments we squeezed aerogel along the magnetic field and filled it up with superfluid ${ }^{3} \mathrm{He}$.

The second important property of NMR in ${ }^{3} \mathrm{He}$ is the stiffness of the wave function, which leads to a mass supercurrent if there is a gradient of the phase of order parameter. In the same way, a gradient of the magnetic part of the wave function leads to a 


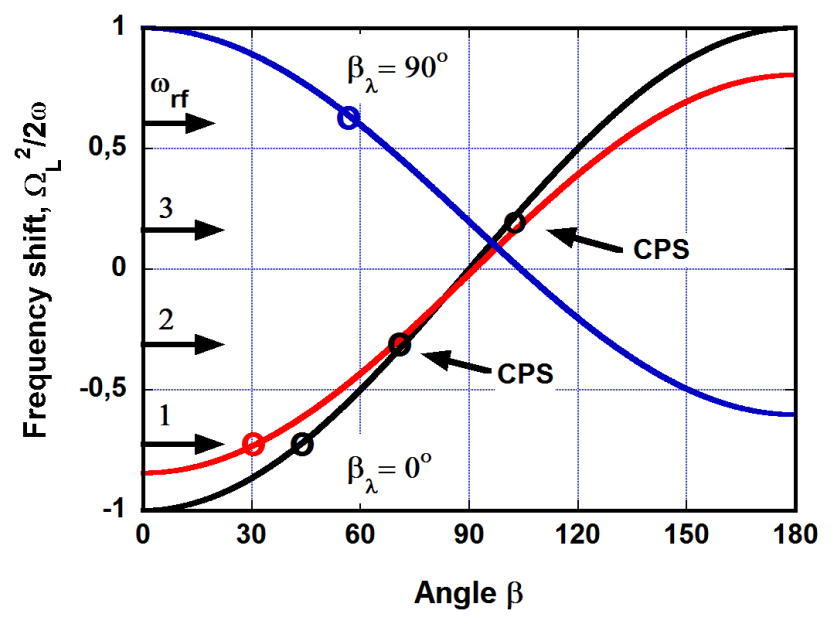

Fig. 1 Formation of CPS in ${ }^{3} \mathrm{He}-\mathrm{A}$ under RF pumping. The lines shows the frequency of NMR as a function of the angle of magnetization deflection in units of maximum frequency shift. The line, marked by $\beta_{\lambda}=90^{\circ}$ shows the frequency for orientation $\mathbf{L} \perp \mathbf{H}$, as usually in the bulk. Other two lines show the frequencies for $\mathbf{L} \| \mathbf{H}$. If one starts continuous wave NMR at frequency 1 and then sweeps it to frequencies 2 and 3, the coherent precession state follows the pumping frequency and the deflection angle increases.

spin supercurrent. Indeed, the property of supercurrent is due to magnetic ordering and can be observed also in solid magnetic ordered materials. For example the spin waves are the result of wave solution with a short distance spin transport. The long distance spin supercurrent was discovered in 1984 in bulk ${ }^{3} \mathrm{He}-\mathrm{B}$ [?]. It is possible in ${ }^{3} \mathrm{He}-\mathrm{B}$ because of a negative feedback on a magnetic inhomogeneity of magnetization, deflected more than $104^{\circ}$. Any inhomogeneity creates a spin current, which flattens the inhomogeneity. As a result the precessing magnetization condenses in a BEC state with a coherent precession of magnetization [?]. On the contrary, in bulk superfluid ${ }^{3} \mathrm{He}-\mathrm{A}$ the coherent precession is unstable as we explain in introduction. An inhomogeneity in the phase of precession leads to a positive feedback by spin, which increases this inhomogeneity. This is a property of most magnetically ordered systems. It also leads to a decreasing frequency with increasing angle of magnetization deflection, as shown in Fig.?? for the branch $\mathbf{L} \perp \mathbf{H}$. The conditions for stable coherent precession and formation of BEC can be realized on the other branch, $\mathbf{L} \| \mathbf{H}$. Particularly, if one sweeps the frequency of RF field up, the coherent precessing state can be formed and moved up, as shown in Fig.??. This experiment was realized in [?] and showed that the all the system follows the frequency of RF field by increasing its angle of deflection.

\section{CW NMR}

We first measured the continuous wave NMR properties of ${ }^{3} \mathrm{He}$ as a function of increasing the RF-field. The transverse magnetization is given by the sum of absorption and dispersion of NMR signal : $M_{\perp}=\sqrt{V_{a b s}^{2}+V_{d i s p}^{2}}$. Results are shown in figure ??. A big non-linear signal appears, which is characteristic of the formation of a coherent 


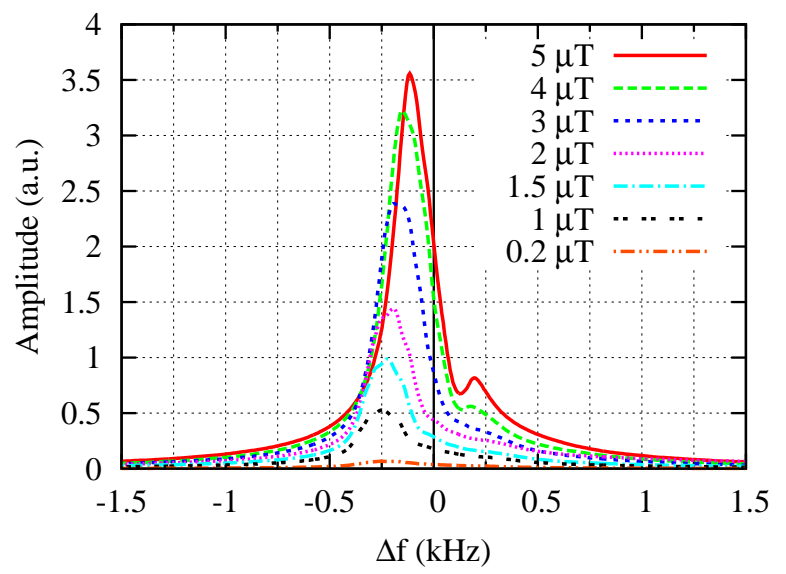

Fig. 2 Continuous wave amplitude with RF-field increasing from 0.2 to $5 \mu \mathrm{T}$. The signal becomes very non linear for high RF-fields. Both the decreasing frequency shift and the big amplitude correspond to coherent precession in A-phase. The small peak near $0.25 \mathrm{kHz}$ might be the signature of HPD in bulk ${ }^{3} \mathrm{He}$ in a $1 \mathrm{~mm}$ channel, near the aerogel sample.

precession state in ${ }^{3}$ He. The frequency shift compared to Larmor frequency decreases as one increases the drive. This corresponds to an increase of the angle of deflection which drives the frequency towards Larmor frequency. The frequency shift for small drive $(0.2 \mu \mathrm{T})$ is about $-250 \mathrm{~Hz}$, and decreases to $-110 \mathrm{~Hz}$ for the maximum drive we applied of $5 \mu \mathrm{T}$. Such a shift corresponds to an angle of deflection near $60^{\circ}$. These results corresponds well to those observed in Kashiva [?]. They are characteristic of the formation of a coherent precession state by continuous wave NMR, as described in [?]. However, in principle, comparable results can be observed in a system of specific nonlinear and non-interacting oscillators, as was shown by V.V. Dmitriev [?]. In this case the coherence is possible due to permanent pumping of external magnetic RF-field.

\section{Pulsed and "switch-off" NMR}

To exclude this scenario, we realized experiments with pulse NMR. We used standard pulse NMR, where a short high power RF-pulse instantaneously deflects magnetization, in this case a RF field of $0.8 \mathrm{mT}$ is applied during a few microseconds. We also used what we will call "switch-off" NMR. In that case, we deflected magnetization with a small RF-field applied by the means of our continuous wave spectrometer. Instead of sweeping the magnetic field, we kept it constant, pumped for approximately one second and observed the free induction decay immediately after switching pumping off. The pumping time is chosen long enough to pump the system in a steady state and short enough to avoid heating. The results of both methods are similar, giving comparable decay times. This decay time is a few times longer than the one we have observed in the normal ${ }^{3} \mathrm{He}$ in the same experimental conditions. It means that at the conditions of pulsed NMR, the state with coherent precession formed itself, without the phasing influence of external RF-field. The "switch-off" NMR gives smoother and bigger amplitude decays as one can see in figure ??. This is an obvious result. During pulsed NMR the magnetic relaxation takes place during the very perturbable time 


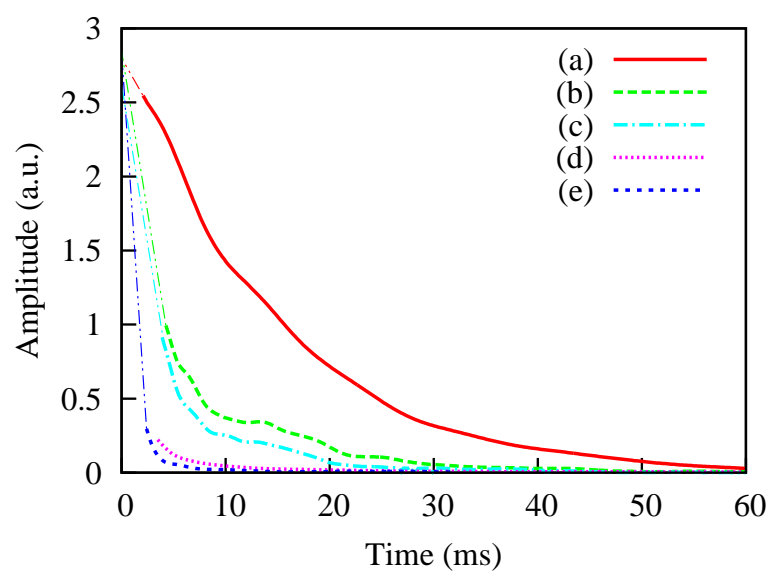

Fig. 3 Comparison of different types of NMR. Curve (a) shows the free induction decay in superfluid A-like phase obtained after switching off pumping on our cw-spectrometer, the initial deflection angle was of about $75^{\circ}$. Curves (b) and (c) show respectively the result of pulse NMR in the superfluid phase for deflection angles of $100^{\circ}$ and $70^{\circ}$. Curves (d) and (e) show respectively the free induction decay for switch off NMR and pulse NMR in normal phase. For each decay, thin lines link the calculated amplitude at $t=0$ to the first point of our fit. The length of decays in superfluid compared to those in normal phase show the existence of a coherent precession state. The difference of amplitudes between curves (d) and (e) is due to the difference in initial precession angles.

of the coherent precession state formation. In continuous wave NMR the coherent precession state is formed before switching off the RF-field and we can see the decay of an equilibrium magnetization.

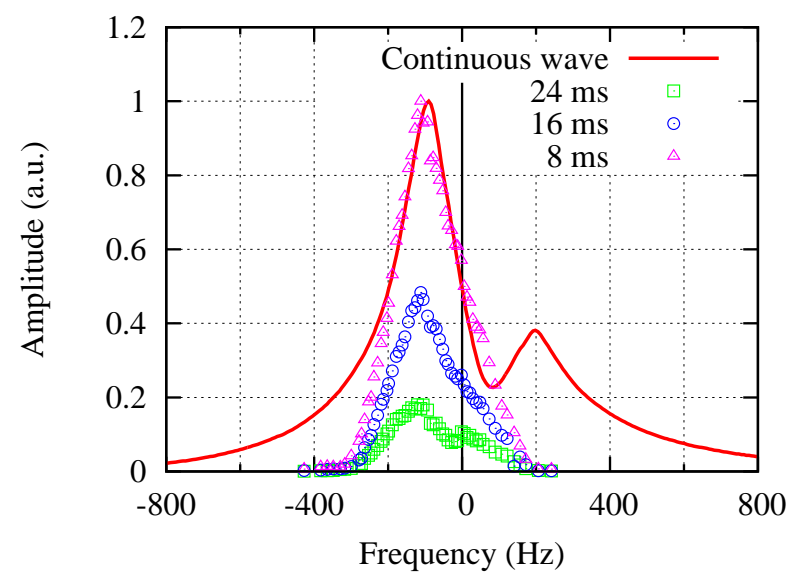

Fig. 4 Comparison of "switch-off" and continuous wave NMR. The continuous line is CW NMR for high drive. Data points represent the amplitude of the free induction decay after 24 , 16 and $8 \mathrm{~ms}$ after switching of the RF pumping at a given frequency. 
Usually, when spins are pumped for a long time, one expects that only those exactly resonating at the pumping frequency will be deflected whereas all other spins will be out of phase inducing signals that cancel each other. However, if a coherent state is excited, all spins in the sample may be deflected and precessing at the pumping frequency. One might see this either in terms of spin supercurrents or in terms of magnon BEC. The decay of the long lived signal we observed is then the signature of the decay of the magnons forming this Bose condensate without loss of coherence.

One can compare the results obtained in "switch-off" NMR with the results of continuous wave experiments. In figure ?? we plot both the CW NMR line at high drive and the amplitude of the free induction decay at various time after switching off the field at the corresponding points of the NMR line. There is a very good agreement between the two types of experiments near the resonance frequency. We see that the wings of the line are an effect of the continuous pumping as they do not appear in "switch-off" NMR.

\section{Conclusion}

In a pulsed NMR experiment we found the formation of coherent precession state which radiates a signal during a $50 \mathrm{~ms}$ after an RF pulse. The duration of the signal in normal ${ }^{3} \mathrm{He}$ at the same magnetic field was about $10 \mathrm{~ms}$. Even more important is the observation of a long ringing signal after switching off the RF field. We created a coherent precessing state signal by CW NMR and then switched of the RF field. We observed a relatively slow decay of a long lived signal with properties which correspond well to the decay of a Bose-Einstein condensate of magnons, formed by CW NMR.

We acknowledge N. Mulders for providing the aerogel samples, and V.V. Dmitriev, I.A. Fomin, M. Kubota, A. Matsubara, T. Mizusaki, and G.E. Volovik for valuable discussions.

\section{References}

1. A.S. Borovik-Romanov, Yu.M. Bunkov, V.V. Dmitriev, et. al., JETP Lett. 40, 1033, (1984); I.A. Fomin, JETP Lett. 40, 1036 (1984).

2. Yu.M. Bunkov and G.E. Volovik, J. Low Temp. Phys. 150, 135-144 (2008).

3. A.S. Borovik-Romanov, Yu.M. Bunkov, V. V. Dmitriev, et. al., JETP Lett. 39, 469-473 (1984).

4. I. A. Fomin, JETP Lett. 30, 164-166 (1979).

5. I. A. Fomin, JETP Lett. 39, 466-469 (1984).

6. Yu. M. Bunkov and G. E. Volovik, Europhys. Lett. 21, 837-843 (1993).

7. T. Kunimatsu, T. Sato, K. Izumina, et.al., JETP Lett. 86, 216 (2007).

8. T. Sato, T. Kunimatsu, K. Izumina, et.al. Phys. Rev. Lett. 101, 055301 (2008).

9. Yu. M. Bunkov and G. E. Volovik, JETP Lett. 89, 356 (2007).

10. G. E. Volovik, JETP Lett. 84, 455 (2006).

11. V.V. Dmitriev, Private communication. 